Schmerz 2013 $27: 356-359$

DOI 10.1007/s00482-013-1341-y

Online publiziert: 1. August 2013

(c) Deutsche Schmerzgesellschaft e.V.

Published by Springer-Verlag Berlin Heidelberg -

all rights reserved 2013

\author{
W. Meißner ${ }^{1,2} \cdot$ F. Petzke ${ }^{3}$ R. Sabatowski ${ }^{4}$ \\ ${ }^{1}$ Sektion Schmerztherapie, Klinik für Anästhesiologie und Intensivmedizin, Universitätsklinikum Jena \\ ${ }^{2}$ Abteilung für Palliativmedizin, Klinik für Innere Medizin II, Universitätsklinikum Jena \\ ${ }^{3}$ Schmerz-Tagesklinik und -Ambulanz, Zentrum Anaesthesiologie, Rettungs- und Intensivmedizin, \\ Universitätsmedizin Göttingen \\ ${ }^{4}$ UniversitätsSchmerzCentrum, Universitätsklinikum Carl Gustav Carus, \\ Technische Universität Dresden
}

\section{Fragen eines lesenden Schmerzmediziners}

Kürzlich wurde die DGS-PraxisLeitlinie „Tumorbedingte Durchbruchschmerzen“ der Deutschen Gesellschaft für Schmerztherapie (DGS) veröffentlicht [1]. Die Notwendigkeit einer eigenen Leitlinienentwicklung wird u. a. damit begründet, dass bestehende Leitlinien wie die zur „Langzeitanwendung von Opioiden bei nicht tumorbedingten Schmerzen (LONTS)“ [2] oder die Nationale VersorgungsLeitlinie (NVL) Kreuzschmerz [3], die u. a. unter Mitwirkung der Deutschen Schmerzgesellschaft entwickelt wurden, weder „den praktischen Erfahrungen engagierter Schmerztherapeuten ... entsprechen, noch die Erwartungen ... Betroffener berücksichtigen" [1]. Ferner wird ihnen Intransparenz, fehlerhafte und inkongruente Wertung der „externen Evidenz“ und „Eminenz-Dominanz" bei der Interpretation der Fakten vorgeworfen, wodurch das Gesamtkonzept von Leitlinien infrage gestellt würde: Es sei „für den praktischen Anwender kaum mehr ersichtlich ..., was (d. h. welche Evidenz) oder wer (d. h. welche Eminenz) in welchem Ausmaß Einfluss genommen hat. Ein inakzeptabler Umstand, der die methodischen Konzepte der Leitlinienerstellung ganz grundsätzlich in Frage stellt, ... “ [1]. Insbesondere werden die Fokussierung auf die Ergebnisse randomisierter, kontrollierter Studien (RCT: „randomized controlled trials“) und „der Einfluss einiger/weniger
Experten“ kritisiert und als eine „unheilige Allianz“ bezeichnet, was „ernsthafte Zweifel an ihrer Sinnhaftigkeit aufkommen lasse“ [1].

Die von der DGS veröffentlichte „neue“ Leitlinienmethodik unterscheidet sich von „konventionellen“ Leitlinien durch eine starke Betonung ,interner“ Evidenz (Erfahrungen praktisch schmerz- und palliativmedizinisch tätiger Ärzte) sowie Patientenbeteiligung als Korrektiv für die „externe“ Evidenz aus randomisierten Studien. Die Empfehlungsbewertungen sollen transparent, klar und eindeutig, handlungsorientiert, leicht verständlich umzusetzen und eindeutig formuliert sein. Damit setze sie „neue Standards für eine patientenorientierte schmerzmedizinische Versorgung auf höchstem Niveau“ [1].

Die PraxisLeitlinie umfasst 7 Kapitel (Definition, Epidemiologie, Diagnostik, klinische Merkmale, Gesundheitsökonomie, Behandlung, Sonstiges) und insgesamt 128 "Empfehlungen", die jeweils mit Angaben zu einem „Evidenzlevel“ (von 1++: „hochwertige Metaanalyse“ bis 4: Expertenmeinung), einem „Evidenzgrad“ (gemeint ist wahrscheinlich „Empfehlungsgrad“; von A: „Die Aussage wird durch mehrere valide klinische Studien oder Metaanalysen ... gestützt“ bis C: „Die Aussage wird nicht durch sichere Studienergebnisse belegt") sowie einem „Konsensgrad Ärzte“ und einem „Konsensgrad Patienten“ versehen sind. Inhaltliche Erläuterungen finden sich mit Ausnahme von Hinweisen auf Literaturstellen nicht.

Erfüllt diese PraxisLeitlinie aber nun die von ihren Herausgebern selbst formulierten Ansprüche, insbesondere vor dem Hintergrund der deutlichen Kritik an Leitlinien der Arbeitsgemeinschaft der Wissenschaftlichen Medizinischen Fachgesellschaften (AWMF), an deren Entwicklung auch die Deutsche Schmerzgesellschaft beteiligt war?

Ein Beispiel: Die Empfehlung VI.21 lautet: „Stark wirksame Opioid-Analgetika der WHO-Stufe III mit schnellem Wirkeintritt und nicht-retardierter Wirkung (sog. ,rapid-onset opioids', [Anm.: ROO - damit sind die transmukosalen Fentanylpräparate gemeint]) sind die Bedarfsmedikation der ersten Wahl für die Behandlung tumorbedingter Durchbruchschmerzen. “Die Empfehlung wird zunächst korrekt mit einem Evidenzlevel 4 („Expertenmeinung“) und folgerichtig einem Evidenzgrad (Empfehlungsgrad?) C („nicht durch sichere Studienergebnisse belegt“) versehen. Der „Konsensgrad Ärzte“ beträgt 99,2\%, derjenige der Patienten 93,3\%. Als Literaturbelege werden 8 Arbeiten (eine kleine klinische Pilotstudie sowie eine Reihe 
narrativer Übersichten, z. T. industriell gesponsert) zitiert. Selbst bei wohlwollender Auslegung sind lediglich 2 Arbeiten als Unterstützung der Empfehlungsaussage zu werten $[4,5]$. Weder die beiden institutionell legitimierten Expertenreviews (durch die Association for Palliative Medicine of Great Britain and Ireland [6] und das „EPCRC opioid guidelines project“ [7]) noch die klinische Studie [8] unterstützen die Empfehlung, ROO generell als Medikation der ersten Wahl bei tumorbedingten Durchbruchschmerzen einzusetzen, sondern bestätigen lediglich deren prinzipielle Eignung. Interessant ist auch: Die vielleicht wichtigste Arbeit, die von der European Association for Palliative Care (EAPC) erarbeiteten „evidence-based recommendations“ zur Opioidbehandlung von Tumorschmerzen, empfiehlt: „In some cases the buccal or intranasal fentanyl preparations are preferable to immediate-release oral opioids.“ Diese Quelle fehlt völlig obwohl bereits Anfang 2012 höchstrangig publiziert [9].

Da es jedoch außer dem Hinweis auf die Literaturstellen keinen Begleit- oder Hintergrundtext gibt, der die Abweichungen zur zitierten Literatur diskutiert, bleibt der Leser irritiert zurück. Mehr noch: Es stellt sich die Frage, welche Weisheit bzw. klinische Erfahrung die 99,2\% „Konsensusärzte“ im Gegensatz etwa zu den nordirischen, EPCRC- bzw. EAPCExperten besitzen. Leider lässt sich dies nicht klären, da nirgendwo angegeben ist, welche und wie viele Ärzte (und Patienten) nach welchen Kriterien beteiligt wurden, insbesondere auch, was diese für die Beteiligung qualifiziert hat.

Allein dieses Beispiel zeigt, dass die PraxisLeitlinie ihrem selbst formulierten (und in anderen Leitlinien verwirklichten) Anspruch nach Transparenz und Nachvollziehbarkeit nicht ansatzweise nachkommt, denn sie fordert selbst: „letztlich sollte es dem für die Umsetzung der Leitlinienempfehlung verantwortlichen Arzt doch ermöglicht werden zu erkennen, ob die Empfehlung für oder gegen ein bestimmtes Verfahren auf der Grundlage nachvollziehbarer Evidenz oder subjektiver Eminenz erfolgte“ [1].

Und auch bei den übrigen Punkten sieht es nicht besser aus:
- Neben klinischen Empfehlungen findet sich eine Vielzahl an allgemeinen Aussagen zu Epidemiologie, Versorgungs- und Qualifizierungsdefiziten, gesundheitsökonomischen und politischen Statements (Regresse, Substitutionspflicht), klinischen Allgemeinplätzen, medizinhistorischen Feststellungen, juristischen Konsequenzen bei unzureichender Versorgung oder Warnungen vor „Pharmakaquotierungen“. Solche Aussagen, die formal genauso gestaltet wurden wie die klinischen Empfehlungen, dürften weltweit einmalig Inhalt einer „Praxis“-Leitlinie sein. Ist das die geforderte „leicht verständlich umsetzbare Handlungsorientierung"?

- Es finden sich an keiner Stelle Angaben $\mathrm{zu}$ Interessenkonflikten der $\mathrm{Au}$ toren und Experten, zur Finanzierung des Projekts oder einer möglichen Unterstützung durch die Industrie bei der Erstellung dieser PraxisLeitlinien - dabei gehört dies zu den minimalen Transparenzanforderungen jeder medizinischen Publikation. Die Auswahl und Anzahl der Konsentierenden bleiben wie erwähnt unklar. Die DGS charakterisiert das methodische Vorgehen der PraxisLeitlinien mit den Worten: „[es] wird auch ein so bislang ... nicht vorhandener Grad an Transparenz erzielt“. Das ist richtig - dieser Transparenzgrad war bisher nicht vorhanden.

- Die PraxisLeitlinien richten sich an „alle schmerztherapeutisch tätigen Ärztinnen und Ärzte“, „an alle Dozenten und Referenten auf dem Gebiet der schmerztherapeutischen Aus, Fort- und Weiterbildung“, , an die Öffentlichkeit“, an „andere ... Fachgesellschaften, sowie ... Herausgeber von Leitlinien“ [1]. Es ist internationaler Konsens, dass ein solcher Anspruch die Einbeziehung von Partnern benachbarter Fachdisziplinen (hier z. B. der Onkologen oder Allgemeinmediziner) voraussetzt und einem transparenten Vorgehen folgt [16]. Ein monodisziplinärer Alleinvertretungsanspruch ist disqualifizierend.

- Es ist unüblich und erschwert den

Zugang, dass eine Leitlinie erst nach einem umständlichen Registrierungsprozess auf einer speziellen Website verfügbar ist. Nicht nur hier sollten sich die Autoren an dem benutzerfreundlichen Vorgehen der AWMF ein Beispiel nehmen.

Damit entpuppt sich die PraxisLeitlinie gerade auch an den Kriterien ihrer Autoren gemessen - als eine Sammlung intransparenter und gerade nicht evidenzbegründeter klinischer Empfehlungen und gesundheitspolitischer Statements, die daher eben keine Entscheidungsunterstützung im klinischen Alltag darstellt.

So ist es nur folgerichtig, dass die DGS mittlerweile gar nicht mehr in Anspruch nimmt, sich an herkömmlichen Kriterien für Leitlinien zu orientieren: „Konsequenterweise kündigen die DGS-PraxisLeitlinien damit dem bisherigen System der wissenschaftlichen Leitlinienmedizin die Gefolgschaft“ [10]. Zum Schluss wird erklärt, es sei nun „genuine Aufgabe der Deutschen Gesellschaft für Schmerztherapie, die für ihr Fachgebiet erforderlichen Standards selbst festzulegen “ [1].

Dies ist umso bedauerlicher, als es ja durchaus berechtigte Kritik und begründeten Verbesserungsbedarf an bestehenden Leitlinien gibt - dies betrifft v. a. die Lesbarkeit, klinische Relevanz und Dissemination. Hier wurde eine Chance vertan.

\section{II}

Vor wenigen Tagen hat die European Medicines Agency (EMA) nach Risikobewertungsverfahren Empfehlungen zum Umgang mit verschiedenen Analgetika publiziert. Neben der Empfehlung, Codein bei Jugendlichen äußerst zurückhaltend und bei Kindern gar nicht einzusetzen, behandeln die beiden anderen Warnungen Diclofenac und Flupirtin. Hinsichtlich Diclofenac wird festgestellt, dass dessen kardiovaskuläre Risiken mit denjenigen der selektiven Cyclooxygenase(COX)-Hemmer vergleichbar und wahrscheinlich höher als bei Ibuprofen liegen. Patienten mit entsprechenden Erkrankungen bzw. Risikofaktoren sollten Diclofenac gar nicht bzw. mit äußerster Vorsicht einnehmen [11].

Das Risikobewertungsverfahren $\mathrm{zu}$ Flupirtin wurde auf Veranlassung des Bundesinstituts für Arzneimittel und Me- 
dizinprodukte (BfArM) eingeleitet, da gehäuft Fallberichte zu Leberwerterhöhungen und Leberschädigungen vorlagen. Darunter finden sich 49 Fälle von Leberversagen einschließlich 12 Fällen mit tödlichem Ausgang sowie 3 Fällen, die zu einer Lebertransplantation führten [12]. Die EMA empfiehlt nun die Anwendung von Flupirtin ausschließlich bei kurz anhaltenden Schmerzen, wenn keine anderen Analgetika wie nichtsteroidale Antirheumatika oder niedrigpotente Opioide eingesetzt werden können, für einen maximalen Zeitraum von 2 Wochen, wobei die Leberwerte wöchentlich kontrolliert werden sollten. Zudem stellt sie fest: „while there were data from studies in the treatment of acute pain, there were insufficient data to support its use in the treatment of long-term pain" [13].

Diese Risikobewertung insbesondere von älteren Substanzen beruht nur zu einem geringen Teil auf RCT, sondern v. a. auf der sorgfältigen Analyse von Versorgungsdaten (insbesondere Registerdaten) sowie von Einzelfällen bzw. Daten nationaler Überwachungsbehörden. Sie macht damit die Limitation von RCT und die Bedeutung von „Alltagsdaten“ deutlich - gleichzeitig zeigt sie jedoch auch die Grenzen des Werts klinischer „Erfahrungen“ auf: Wohl wenige Kliniker, die Autoren nehmen sich hier nicht aus, werden die nun wahrscheinlichen Unterschiede der Risikoprofile von Diclofenac und Ibuprofen allein aus ihrer klinischen Expertise - und sei sie noch so langjährig - wahrgenommen haben. Argumente wie „Da ist seit 20 Jahren nichts passiert“ oder „Damit habe ich gute Erfahrungen gemacht" sind vollkommen untauglich, insbesondere wenn es um seltene, aber lebensbedrohliche Ereignisse geht.

Etwas anders ist die Situation bei Flupirtin: Leberwerterhöhungen und Fälle von Leberschädigung waren seit Langem bekannt und wurden in der schmerztherapeutischen Szene mit gewisser Besorgnis diskutiert. Denn neben den erwähnten Fallberichten liegen hierzu auch Daten aus prospektiven Studien vor: So kam es bei 3\% nach 1-wöchiger [14] bzw. bei 31\% der Patienten nach 6-wöchiger Flupirtinbehandlung zu Leberwerterhöhungen, was zum Studienabbruch führte [15]. Daher ist es vielleicht nachvollziehbar, dass noch kürzlich die Herstellerfirma (vergeblich) gegen die Nichtempfehlung von Flupirtin in der NVL „Kreuzschmerz“ klagte. Schwer verständlich ist aber, dass auch einige Schmerzexperten in diesen Chor einfielen und der NVL unterstellten, dadurch die Patientenversorgung zu verschlechtern. „Primum non nocere“?

III

Was aber haben beide Fälle miteinander zu tun? Sowohl die PraxisLeitlinien als auch die EMA-Empfehlungen verdeutlichen und bekräftigen die überragende Bedeutung der evidenzbasierten Medizin (EBM), die - zumindest besser als jede Alternative - in der Lage ist, uns Kliniker in der Entscheidungsfindung zu unterstützen, unsere klinische Erfahrung zu ergänzen, Transparenz zu erhöhen und Interessenkonflikte sichtbar und kalkulierbar zu machen. Dies funktioniert jedoch nur, wenn die EBM im Sinne seiner „Erfinder" angewendet und nicht je nach Gefallen oder Nichtgefallen ihrer Ergebnisse „Zurechtgebogen“" oder selektiv gehandhabt wird.

Dies bedeutet nicht, dass die EBM nicht verbesserbar ist: Die - auch von Mitgliedern der DGS geäußerten - Kritikpunkte an manchen Aspekten der EBM inklusive der daraus entstandenen Leitlinien sind durchaus berechtigt und sollten gemeinsam mit anderen Fachverbänden und nicht zuletzt auch mit der AWMF diskutiert werden. Zunehmend kritisch wird auch in der „Hardcore-EBM-Szene“ mittlerweile die Dominanz der doppelblinden RCT und systematischen Metaanalysen gegenüber anderen Formen des Erkenntnisgewinns diskutiert. Insbesondere Versorgungs- und Registerdaten, die bestimmte Qualitätskriterien erfüllen, sollten in Zukunft einen höheren Stellenwert bekommen. Weitere Probleme umfassen Aspekte der klinischen Relevanz, Übertragbarkeit auf den Alltag und praktischen Umsetzung.

Dennoch: Nur die kritische und stetige Weiterentwicklung der EBM - aber nicht ihre Relativierung - wird dazu beitragen, unsere Medizin wirkungsvoller, sicherer und angemessener zu gestalten. Der Weg dahin ist mühsam - aber einen anderen gibt es nicht.

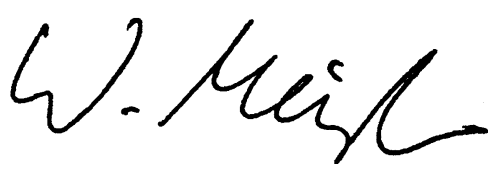

W. Meißner

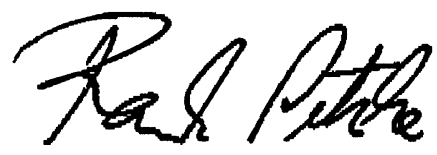

F. Petzke

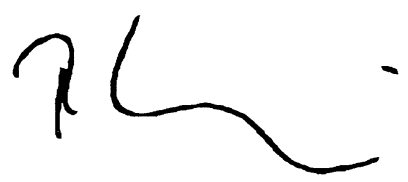

R. Sabatowski

\section{Korrespondenzadresse}

Prof. Dr. W. Meißner

Sektion Schmerztherapie, Klinik für

Anästhesiologie und Intensivmedizin,

Universitätsklinikum Jena

07740 Jena

winfried.meissner@med.uni-jena.de

\section{Einhaltung der ethischen Richtlinien}

Interessenkonflikt. W. Meißner: Forschungsunterstützung: Fakultät, EU; klinische Auftragsforschung: Cephalon, GWPharma; Vortragstätigkeit: MSD, Mundipharma, Grünenthal, Pfizer, Cephalon, Shire; Beratertätigkeit: Mundipharma, Grünenthal, QRxPharma, Medicines Company. F. Petzke: Vortragstätigkeit: JansenCilag, Eli-Lilly; Beratertätigkeit: Jansen-Cilag, Grünenthal. R. Sabatowski: klinische Auftragsforschung: Grünenthal, Allergan, Astellas; Vortragstätigkeit: MSD; Beratertätigkeit: Jansen-Cilag, Grünenthal.

\section{Literatur}

1. Deutsche Gesellschaft für Schmerztherapie (DGS) (2013) PraxisLeitlinie. Tumorbedingte Durchbruchschmerzen. http://www.dgs-praxisleitlinien.de/

2. Arbeitsgemeinschaft der Wissenschaftlichen Medizinischen Fachgesellschaften (AWMF) (2009) Langzeitanwendung von Opioiden bei nicht tumorbedingten Schmerzen (LONTS). http://www.awmf. org/leitlinien/detail/ll/041-003.html

3. Nationale VersorgungsLeitlinie (NVL) Kreuzschmerz. http://www.kreuzschmerz.versorgungsleitlinie.de

4. Casuccio A, Mercadante S, Fulfaro F (2009) Treatment strategies for cancer patients with breakthrough pain. Expert Opin Pharmacother 10:947953

5. Lossignol DA, Dumitrescu C (2010) Breakthrough pain: progress in management. Curr Opin Oncol 22:302-306 
6. Davies AN, Dickman A, Reid C et al (2009) The management of cancer-related breakthrough pain: recommendations of a task group of the Science Committee of the Association for Palliative Medicine of Great Britain and Ireland. Eur J Pain 13:331338

7. Zeppetella G (2011) Opioids for the management of breakthrough cancer pain in adults: a systematic review undertaken as part of an EPCRC opioid guidelines project. Palliat Med 25:516-524

8. Zeppetella G (2008) Opioids for cancer breakthrough pain: a pilot study reporting patient assessment of time to meaningful pain relief. J Pain Symptom Manage 35:563-567

9. Caraceni A, Hanks G, Kaasa S et al (2012) Use of opioid analgesics in the treatment of cancer pain: evidence-based recommendations from the EAPC. Lancet Oncol 13:e58-68

10. Überall M (2013) Die DGS-Praxisleitlinie tumorbedingte Durchbruchschmerzen. Schmerzmedizin 29:7-8

11. European Medicines Agency (2013) New safety advice for diclofenac - CMDh endorses PRAC recommendation. http://www.ema.europa.eu

12. Bundesinstitut für Arzneimittel und Medizinprodukte (BfArM) (2013) http://www.bfarm.de/DE/ Pharmakovigilanz/risikoinfo/2013/Rl-flupirtin.html

13. European Medicines Agency (2013) Restrictions in the use of flupirtine-containing medicines - CMDh endorses PRAC recommendation. http://www. ema.europa.eu

14. Li C, Ni J, Wang Z et al (2008) Analgesic efficacy and tolerability of flupirtine vs. tramadol in patients with subacute low back pain: a double-blind multicentre trial*. Curr Med Res Opin 24:35233530

15. Michel MC, Radziszewski P, Falconer C et al (2012) Unexpected frequent hepatotoxicity of a prescription drug, flupirtine, marketed for about 30 years. Br J Clin Pharmacol 73:821-825

16. http://www.awmf.org/leitlinien/awmf-regelwerk. html
R. Larsen

\section{Anästhesie und Intensivmedizin für die Fachpflege}

unter Mitarbeit von T. Müller-Wolff

Heidelberg: Springer-Verlag GmbH 2012,

8. Auflage, 1030 S., 412 Abb.,

(ISBN 978-3-642-28290-4), 49.95 EUR

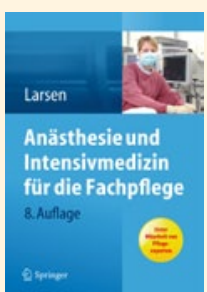

Mit der 8. Auflage des

Standardwerks „Anästhesie und Intensivmedizin für die Fachpflege" legen Herr Professor Larsen und sein Mitherausgeber auf dem pflegerischen Bereich, Herr Müller-Wolff, ein Lehrbuch und Nachschlagewerk vor, das sowohl Einsteigern als auch dem wachsenden Anforderungsprofil an die Fachkrankenpflege gerecht wird. Auf über 1.000 Seiten werden dem Leser die pflegerelevanten Themen aus Anästhesie und Intensivmedizin systematisch und umfassend erschlossen. Von den Grundlagen der Anästhesie und Intensivmedizin aus werden spezielle Themen folgerichtig aufgegriffen und in einem für den Leser gut erfassbaren, inhaltlich aber trotzdem anspruchsvollen Niveau aufgearbeitet. Dabei eignet sich das Buch nicht nur als Nachschlagewerk für die erfahrene Fachpflegekraft, vielmehr wird das Buch auch dem Neueinsteiger in der Intensivpflege und Anästhesie gerecht, indem ein stufenweises Herangehen an Anästhesie und Intensivmedizin eröffnet wird.

Die didaktische Aufarbeitung mit vielen praxisnahen Abbildungen und Tabellen erleichtert das Verständnis der praktischen Abläufe; vertiefend ebenso auch der physiologischen und pharmakologischen Hintergründe. Daneben werden zu allen Themen Praxistipps gegeben, die die Umsetzung von der Theorie in die Praxis erleichtern und Fallstricke vermeiden. Wer dann noch Fragen hat, kann sich anhand der Leseempfehlungen in vertiefende Literatur oder Internetquellen einarbeiten. Von der Vorbereitung des Anästhesiearbeitsplatzes bis zur leitliniengerechten Versorgung Schwerverletzter und zu Besonderheiten nach Herzoperationen wird die komplette Bandbreite des Wissens für die Anästhesie- und Intensivpflege leicht verständlich präsentiert. So wird eine optimale Vorbereitung auf die Herausforderungen der nächsten Schicht am Patienten oder für die bevorstehende Prüfung erreicht. Neben der Einarbeitung aktueller insbesondere auch pflegerelevanter Leitlinien für die Patientenbehandlung wie z.B. der S3Leitlinien Analgosedierung, Delirbehandlung und Schmerztherapie wurde ein besonderes Augenmerk in dieser Auflage darauf verwendet, die pflegerischen Aufgabenbereiche noch besser zu vermitteln. Durch ausgewiesene Pflegeexperten werden für den Leser wichtige praktische und berufsethische Aspekte des Handelns am Patienten beleuchtet und diese in anschauliche und praktisch umsetzbare Abbildungen und Arbeitsanleitungen gefasst. Erweiterte Verantwortungs- und Aufgabenbereiche insbesondere in der Intensivpflege, Delegation ärztlicher Maßnahmen und neue Arbeitsteilungen zwischen unterschiedlichen Berufsgruppen verlangen professionelle Herangehensweisen, um eine optimale Behandlung und Pflege der Patienten zu ermöglichen. Herr Professor Larsen hat nun sein bewährtes Standardwerk gemeinsam mit seinem pflegerischem Herausgeber in der 8 . Auflage der Verzahnung des multiprofessionellen Wissens verschrieben und bereitet den Leser damit bestens auf die anspruchsvolle Arbeit am Patienten vor.

Fazit: Larsens „Anästhesie und Intensivmedizin für die Fachpflege" arbeitet das notwendige Wissen für die Pflege systematisch, praxisrelevant, zielgruppengerecht und gut lesbar auf. Durch die grundlegende Überarbeitung unter Einbeziehung von Pflegeexperten und weiteren Pflegethemen gewinnt das Werk mit seiner 8 . Auflage deutlich und sollte in keinem Bücherregal in der Anästhesie- und Intensivpflege fehlen.

Professor Dr. Axel R. Heller (Dresden) 